\title{
Effects of yoga on depressive symptoms in people with mental disorders: a systematic review and meta- analysis
}

\author{
Jacinta Brinsley (D) ,' Felipe Schuch, ${ }^{2}$ Oscar Lederman, ${ }^{3}$ Danielle Girard, ${ }^{1}$ \\ Matthew Smout, ${ }^{4}$ Maarten A Immink, ${ }^{1}$ Brendon Stubbs, ${ }^{5}$ Joseph Firth, ${ }^{6}$ Kade Davison, ${ }^{1}$ \\ Simon Rosenbaum (1D) 7,8
}

- Additional material is published online only. To view please visit the journal online (http://dx.doi.org/10.1136/ bjsports-2019-101242).

For numbered affiliations see end of article.

Correspondence to Jacinta Brinsley, School of Health Sciences, University of South Australia, Adelaide, SA 5001, Australia;

jacintabrinsley.aep@gmail.com

Accepted 19 March 2020 Published Online First 18 May 2020
Check for updates

(C) Author(s) (or their employer(s)) 2021. No commercial re-use. See rights and permissions. Published by BMJ.

To cite: Brinsley J,

Schuch F, Lederman 0 ,

et al. Br J Sports Med

2021:55:992-1000.

\section{ABSTRACT}

Objective To assess whether physically active yoga is superior to waitlist control, treatment as usual and attention control in alleviating depressive symptoms in people with a diagnosed mental disorder recognised by the Diagnostic and Statistical Manual of Mental Disorders (DSM).

Design Systematic review and meta-analysis following the Preferred Reporting Items for Systematic Reviewsand Meta-Analyses (PRISMA) guidelines.

Data sources Data were obtained from online databases (MEDLINE, EMBASE, PsychINFO, CENTRAL, EMCARE, PEDro). The search and collection of eligible studies was conducted up to 14 May 2019 (PROSPERO registration No CRD42018090441).

Eligibility criteria for selecting studies We included randomised controlled trials with a yoga intervention comprising $\geq 50 \%$ physical activity in adults with a recognised diagnosed mental disorder according to DSM-3, 4 or 5 .

Results 19 studies were included in the review (1080 participants) and 13 studies were included in the metaanalysis (632 participants). Disorders of depression, post-traumatic stress, schizophrenia, anxiety, alcohol dependence and bipolar were included. Yoga showed greater reductions in depressive symptoms than waitlist, treatment as usual and attention control (standardised mean difference $=0.41 ; 95 \% \mathrm{Cl}-0.65$ to -0.17 ; $p<0.001)$. Greater reductions in depressive symptoms were associated with higher frequency of yoga sessions per week $(\beta=-0.44, p<0.01)$.

\section{INTRODUCTION}

Globally, mental disorders are responsible for $32.4 \%$ of disability adjusted life years (a year of 'healthy' life lost), placing mental disorders at a distant first in the global burden of disease in terms of years lived with disability. ${ }^{1}$ Depressive disorders, such as major depressive disorder, are the leading cause of disability worldwide, affecting more than 340 million people. ${ }^{2}$ They are predicted to be the second largest contributor to the global burden of disease by the year $2020 .^{3}$ Depressive disorders are highly comorbid with other mental disorders, such as anxiety disorders ${ }^{4}$ and psychotic disorders. ${ }^{5}$ Physical comorbidities are also present in $65 \%$ of people with depressive disorders, ${ }^{6}$ commonly including obesity, type 2 diabetes, metabolic syndrome and cardiovascular disease. ${ }^{7}$ This is driven in part by modifiable lifestyle related risk factors, such as low levels of physical activity and high levels of sedentary behaviour. ${ }^{8}$ Physical inactivity is associated with higher levels of depressive symptoms in the general population ${ }^{9}$ and in those with major depressive disorder. ${ }^{10}$ There is a clear need for interventions which are efficacious in improving both physical activity and depressive symptoms ${ }^{1112}$ and multicomponent lifestyle interventions incorporating a combination of physical activity, exercise and diet. ${ }^{1314}$ Treatment guidelines for mental disorders from leading international organisations now recommend the integration of physical activity based interventions as part of routine psychiatric care. ${ }^{11}{ }^{15-17}$ Despite these recommendations, translation of evidence into clinical practice and routine implementation of exercise interventions as part of standard care is limited. ${ }^{18}$

One form of exercise that is increasing in popularity in western culture, particularly among psychiatric patients, ${ }^{19}$ is yoga. Yoga is a multicomponent mind-body practice comprising physical postures, movement, breathing control and techniques, relaxation, mindfulness and meditation. ${ }^{20}$ Mindfulness, meditation and exercise are all effective in reducing depressive symptoms. ${ }^{21} 22$ Yoga practice often includes a combination of each and therefore may have additional benefits beyond a single component (eg, mindfulness, meditation or exercise alone). Furthermore, yoga has demonstrated efficacy in improving exercise adherence and may provide an alternative engagement strategy for people who do not want to engage in conventional forms of exercise. ${ }^{23}$

People living with mental disorders commonly experience depressive symptoms-for example, $25 \%$ of people diagnosed with schizophrenia and $81 \%$ of people diagnosed with generalised anxiety disorder also have depression. ${ }^{2425}$ Several systematic reviews and meta-analyses have assessed a range of yoga interventions, using various styles of yoga, and have shown improvements in mental health across various mental disorders ${ }^{26}{ }^{27}$ (see table 1 ). However, no previous review has investigated the transdiagnostic benefits of yoga. Earlier reviews that focused on yoga for people with depression reported positive effects but all conclude that findings must be interpreted with caution due to the heterogeneity of the yoga interventions and poor methodological reporting. ${ }^{28-30}$ This review differs from previous reviews as it includes a range of mental disorder 
Table 1 Summary of previous literature reviews on yoga and mental disorders

\begin{tabular}{|c|c|c|c|c|c|c|c|c|}
\hline Author & Year & $\begin{array}{l}\text { Systematic } \\
\text { review }\end{array}$ & $\begin{array}{l}\text { Meta- } \\
\text { analysis }\end{array}$ & Population & $\begin{array}{l}\text { Assessment } \\
\text { of risk of } \\
\text { bias }\end{array}$ & $\begin{array}{l}\text { DSM/ICD } \\
\text { diagnostic } \\
\text { criteria }\end{array}$ & $\begin{array}{l}\text { Reference } \\
\text { to PRISMA } \\
\text { statement }\end{array}$ & General result \\
\hline Balasubramaniam et $a l^{68}$ & 2013 & Yes & No & $\begin{array}{l}\text { Varied psychiatric } \\
\text { disorders }\end{array}$ & Yes & No & Yes & $\begin{array}{l}\text { High quality evidence for positive effect on depression } \\
\text { and sleep }\end{array}$ \\
\hline Broderick et al ${ }^{69}$ & 2015 & No & No & Schizophrenia & Yes & Yes & Yes & Evidence too weak \\
\hline Cramer et $\left.a\right|^{26}$ & 2013 & Yes & Yes & Schizophrenia & Yes & Yes & Yes & $\begin{array}{l}\text { Moderate evidence for positive short term effect on } \\
\text { quality of life }\end{array}$ \\
\hline Cramer et $a l^{70}$ & 2017 & Yes & No & Depression & Yes & Yes & Yes & $\begin{array}{l}\text { Comparable effects of yoga compared with exercise and } \\
\text { medication }\end{array}$ \\
\hline Cramer et $a l^{71}$ & 2018 & Yes & Yes & PTSD & Yes & No & Yes & $\begin{array}{l}\text { Weak recommendation for yoga as an adjunctive } \\
\text { treatment }\end{array}$ \\
\hline Cramer et $a l^{72}$ & 2018 & Yes & Yes & Anxiety & Yes & No & Yes & $\begin{array}{l}\text { Moderate evidence for medium-large effect compared } \\
\text { with no treatment and active comparator control }\end{array}$ \\
\hline Cramer et $a l^{28}$ & 2013 & Yes & Yes & Depression & Yes & Yes & Yes & $\begin{array}{l}\text { Limited to moderate evidence for improvements in } \\
\text { depression and anxiety }\end{array}$ \\
\hline Da Silva et $a l^{73}$ & 2009 & No & No & $\begin{array}{l}\text { Varied psychiatric } \\
\text { disorders }\end{array}$ & No & No & No & Moderate evidence for positive effect on mood disorders \\
\hline Gallegos et $\mathrm{al}^{74}$ & 2017 & No & Yes & PTSD & Yes & No & No & Small to moderate effect on PTSD symptoms \\
\hline Gong et $a^{60}$ & 2015 & Yes & Yes & Prenatal depression & No & No & No & $\begin{array}{l}\text { Low-moderate quality evidence for reducing depressive } \\
\text { symptoms }\end{array}$ \\
\hline Kirkwood et al27 & 2005 & Yes & No & Anxiety & No & No & No & High quality evidence for positive effect on $O C D$ \\
\hline Pilkington et $a^{29}$ & 2005 & No & No & Depression & No & No & No & Evidence too weak \\
\hline Uebelacker et $a l^{30}$ & 2010 & No & No & Depression & No & No & No & Evidence too weak \\
\hline Vancampfort et $a l^{75}$ & 2012 & Yes & No & Schizophrenia & No & Yes & Yes & $\begin{array}{l}\text { Moderate evidence for improved psychopathology, } \\
\text { positive and negative symptoms, HRQL }\end{array}$ \\
\hline
\end{tabular}

diagnoses and considers the transdiagnostic potential for yoga across all mental disorder groups, thus allowing for a more comprehensive assessment of the potential benefits for depressive symptoms across the range of mental disorders.

Hence the primary aim of this review was to determine the effects of physically active yoga on depressive symptoms compared with control in people with any type of mental disorder. Additionally, the review examines the physical health outcomes of yoga interventions for people with mental disorders. We also sought to identify the key components of yoga based interventions that are associated with better outcomes.

\section{METHODS}

This systematic review and meta-analysis was conducted according to the Preferred Reporting Items for Systematic Reviewsand Meta-Analyses (PRISMA) guidelines ${ }^{31}$ and was prospectively registered with the PROSPERO database (ID: CRD42018090441).

\section{Data sources and searches}

The following sources were searched from database inception to 14 May 2019: MEDLINE (Ovid), EMBASE (Ovid), PsychINFO (Ovid), the Cochrane Central Register of Controlled Trials (CENTRAL), EMCARE (Ovid) and the Physiotherapy Evidence Database (PEDro). MeSH headings used were 'mental disorders' or 'mental disease' AND 'yoga', with all branches entered as keywords (Supplementary Table 1). Manual searches were conducted using the reference lists from recovered articles and relevant systematic reviews and meta-analyses.

\section{Study selection/inclusion criteria Participants}

Studies of adults aged 18 years or older with a current Diagnostic and Statistical Manual of Mental Disorders (DSM)-3, 4 or $5^{32}$ diagnosis of any mental disorder, including depressive disorders, anxiety disorders, affective disorders, trauma and stress related disorders, psychotic disorders, panic disorders and alcohol and substance use disorders were included. Participants with other clinical health conditions in addition to a mental disorder were not excluded.

\section{Interventions}

Yoga interventions were defined as a structured, formal and premeditated form of physical activity involving the integration of specific body movements (asana) with breathing (pranayama) and/or mindfulness (including meditation), ${ }^{27}$ where the movement component (physical activity) made up more than $50 \%$ of the total intervention.

\section{Control conditions}

Treatment as usual, waitlist or attention controls were included. Trials that compared one type of physical activity with another (eg, yoga vs aerobic exercise) without an adequate control group were excluded. ${ }^{33}$

\section{Outcome measures}

The primary outcome measure was change in depressive symptoms across all mental disorders (mean \pm SD), measured on validated scales, scored from baseline to post-intervention. Secondary outcomes of interest were exercise capacity (eg, measures of cardiorespiratory fitness and strength), biomarkers (eg, cortisol), quality of life, cognition, sleep, anthropometry (eg, body mass index, waist circumference), remission rates, attendance, dropout rates and adverse events.

\section{Study design}

Only randomised controlled trials (RCTs) were eligible.

\section{Exclusion criteria}

In the case of multiple publications from the same study, outcome measures were collated to create a 'complete study'. Trials were 
not excluded because of nationality or clinical setting (inpatient, outpatient, community setting or mixed). Studies not available in English were excluded.

\section{Data extraction}

After removal of duplicates, two researchers (JB and OL) independently screened titles and abstracts using Covidence (Covidence systematic review software, Veritas Health Innovation, Melbourne, Australia). Both authors applied eligibility criteria, and a final list of included articles was developed through consensus. A third reviewer (SR) was available for mediation throughout this process.

Data were extracted from the included articles using a predetermined data extraction form by two authors (JB and DG) and cross-checked for accuracy by a third author (OL). The data extracted included participant and trial characteristics (diagnosis, type of yoga, control condition, programme length, session duration, frequency, intensity, supervision) and yoga effects on mental disorder severity, as per disorder specific scales. Information regarding the yoga interventions was extracted for meta-regression analyses. The type of yoga was determined by the authors' specifications in the protocol. A simple 'yes', 'no' or 'not specified' was used to determine whether the following aspects of yoga were reported in the intervention: specific yoga postures, consistent sequencing of postures, yogic philosophy, meditation, mindfulness ('attention to breath' met this criterion), breathing techniques ('pranayama' or other controlled breathing exercises met this criterion) and/or encouragement of home practice.

\section{Study quality}

Study quality was reported using the PEDro checklist ${ }^{34}$ and scores as reported on the PEDro database. Two authors crosschecked $35 \%$ of the included studies against PEDro scoring for quality assurance. A study was deemed to be of high quality with a score of $6-10$, of fair quality with a score of $4-5$ and of low quality with a score $<4$. The PEDro scores for articles included in this review are presented in table 2.

\section{Data analysis}

Random effects meta-analysis was conducted with Comprehensive Meta-Analysis software (CMA, V.3) in the following steps. Firstly, changes in mental disorder symptoms were calculated using pre- and post-means (SD) of depressive symptom severity scores of the yoga and control groups, calculating the standardised mean difference (SMD) and 95\% CI as the effect size. Secondly, subgroup analyses of yoga modality (eg, vinyasa, chair, kripalu), diagnoses, intervention supervision (yes vs home practice) and intensity, as specified by the authors (eg, gentle hatha) were conducted. In the final step, exploratory meta-regression analyses investigating the moderating role of trial duration, number of sessions per week, trial quality, session length and number of components of yoga used in the intervention on the effect size were conducted. In the absence of sufficient data to conduct meta-analyses, a narrative synthesis of results was conducted. Statistical heterogeneity was assessed using the $\mathrm{I}^{2}$ statistic. An $\mathrm{I}^{2}$ value $>75 \%$ was considered to indicate considerable heterogeneity, $\mathrm{I}^{2} 50-75 \%$ was considered to indicate substantial heterogeneity and $\mathrm{I}^{2}<40 \%$ was considered to indicate limited heterogeneity. ${ }^{35}$ Publication bias was assessed with Egger $^{36}$ and Begg and Mazumdar, ${ }^{37}$ and corrected using Duval and Tweedie trim and fill analysis. ${ }^{38}$
RESULTS

Search results and flow of trials through the review

A total of 3880 records (excluding duplicates) were identified, and the titles and abstracts were screened. Full text versions were retrieved for 80 articles, of which 19 were eligible for inclusion. Thirteen of those reported changes in depressive symptoms and were included in the meta-analysis. Six studies were excluded from the quantitative analyses as they did not report depression symptom scores. Figure 1 shows the flow of studies through the review and reasons for exclusion.

\section{Characteristics of included trials}

Characteristics of participant sample, primary diagnosis, diagnostic criteria, yoga intervention and control group are shown in table 3. Analyses of physical health outcomes were not performed because of insufficient data.

Of the 19 included RCTs, 12 originated from the USA, ${ }^{19} 39-49$ two from India, ${ }^{50} 51$ two from Japan ${ }^{52} 53$ and one each from China, ${ }^{54}$ Germany ${ }^{55}$ and Sweden. ${ }^{56}$ Nine trials included patients with a depressive disorder (antenatal $=5^{39-414347}$; bipolar depression $=1^{48}$; major depressive disorder $=3^{444655}$ ), five included a diagnoses of schizophrenia, ${ }^{19-5257}$ three with a post-traumatic stress disorder (PTSD) diagnosis, ${ }^{42} 4549$ one with diagnosed alcohol dependence ${ }^{56}$ and one with a range of psychiatric disorders. ${ }^{53}$ A total of 1080 participants were included across the 19 RCTs; sample sizes of individual studies ranged from 18 to 122 (median $=57$; mean $=57$ ), with $69 \%$ of the total sample being women.

Nine studies ${ }^{39-41} 4345-4855$ reported using the Structured Clinical Interview for DSM for diagnostic purposes. One study used the Mini International Neuropsychiatric Interview, ${ }^{44}$ one used the Clinician Administered PTSD Scale for DSM ${ }^{49}$ and another used scores above 57 on the PTSD Checklist for DSM. ${ }^{42}$ One study used the Structured Clinical Interview for the Positive and Negative Syndrome Scale (PANSS), ${ }^{19}$ two studies used the International Classification of Diseases- $10,{ }^{52} 53$ two studies used psychiatrist interviews to confirm DSM criteria ${ }^{5051}$ and two studies did not specify how the diagnosis was made. ${ }^{5456}$ Tools used to measure changes in depression symptoms were the Quick Inventory of Depressive Symptomatology (QIDS SR-16), ${ }^{46-48}$ Depression Anxiety and Stress Scale (DASS-21), ${ }^{42}$ Centre for Epidemiologic Studies Depression Scale (CES-D), ${ }^{40} 4158$ Hamilton Depression Rating Scale (HAM-D/HDRS), ${ }^{39} 55$ Beck Depression Inventory (BDI), ${ }^{44}$ Hospital Anxiety and Depression scale (HAD) ${ }^{56}$ PANSS Depression subscale ${ }^{19}$ and the Calgary Depression Scale (CDS). ${ }^{54}$

\section{Interventions}

In total, $\mathrm{n}=578$ participants were assigned to yoga and $\mathrm{n}=502$ to control conditions. Seven RCTs utilised attention control groups, including health education, ${ }^{43} 464749$ yoga education, ${ }^{44}$ social support ${ }^{40}$ and bibliotherapy. ${ }^{48}$ Seven RCTs utilised waitlist control ${ }^{19} 394245505159$ and five utilised treatment as usual. ${ }^{4152535556}$ Yoga interventions were on average 2.4 months long (range 1.5-2.5 months) with 1.6 sessions per week (range 1-3 sessions) of a duration of $60 \mathrm{~min}$ (range 20-90 min). Types of yoga were hatha, ${ }^{44} 464849525355$ vinyasa, ${ }^{39}$ SVYASA (Swami Vivekananda Yoga Anusandhana Samsthana), ${ }^{50}{ }^{51}$ kundalini, ${ }^{42}$ kripalu $^{45}$ or were unspecified. ${ }^{19404143475456}$ Seventeen interventions were supervised by yoga practitioners ${ }^{19} 39-4952-56$ and two interventions were primarily unsupervised as they were home practices. ${ }^{5051}$ 


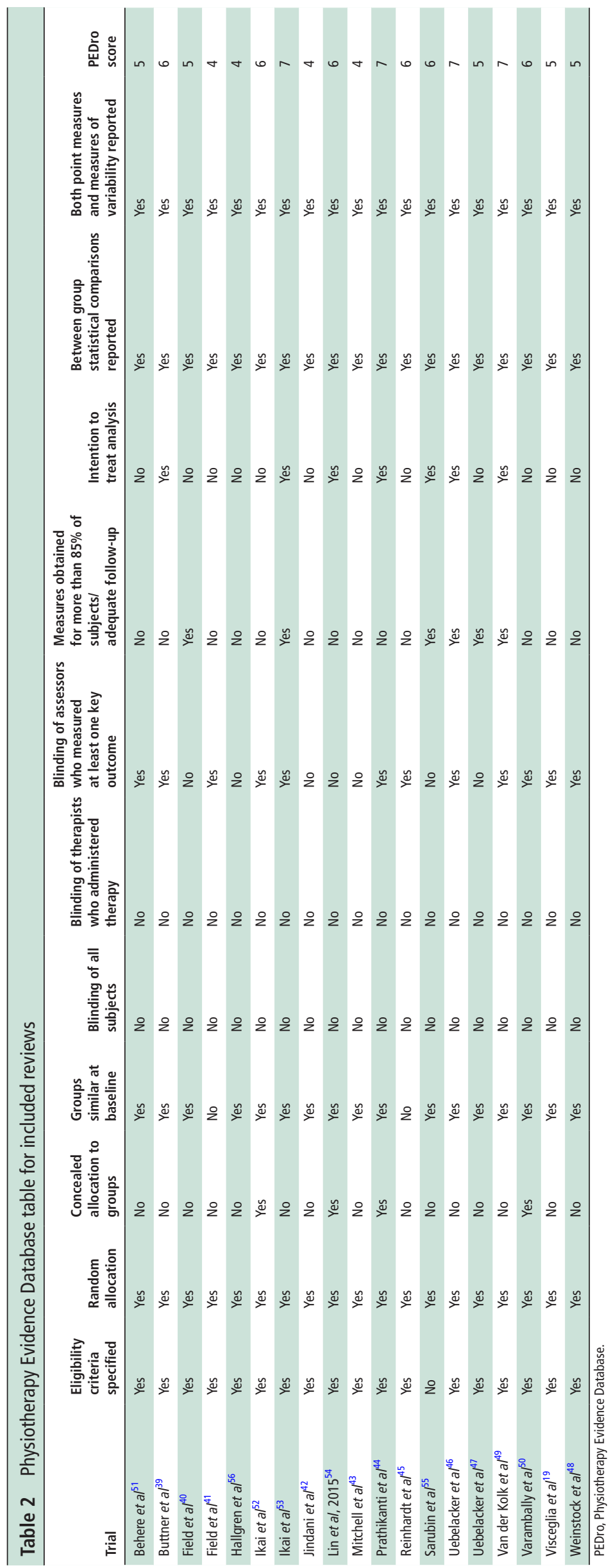

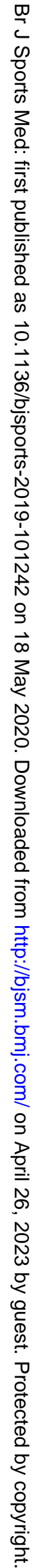




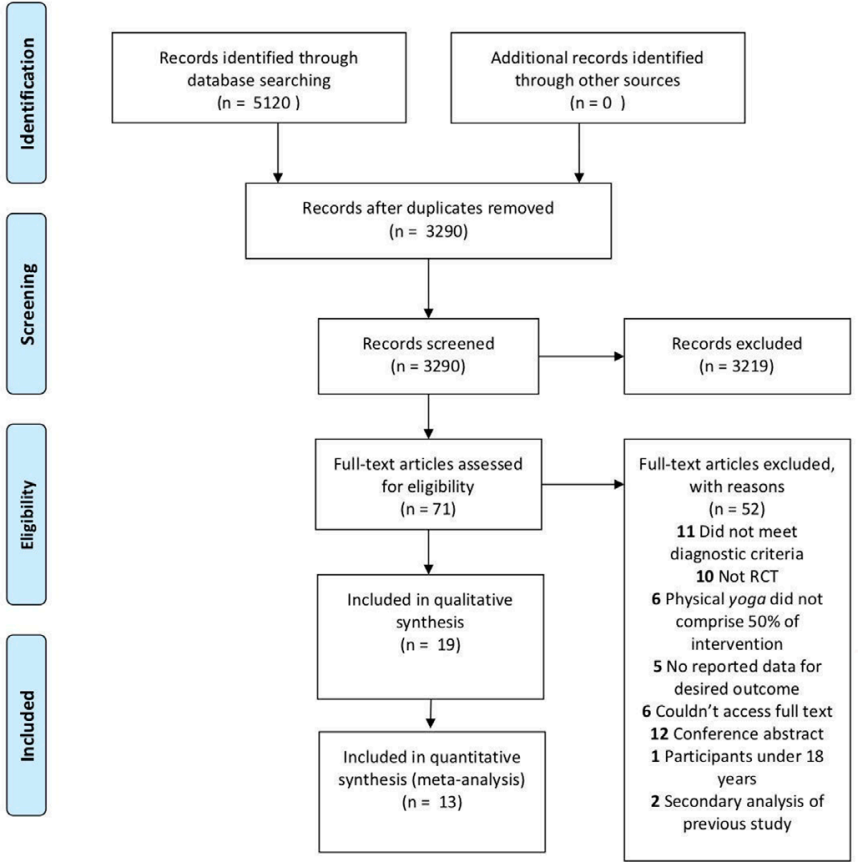

Figure 1 Identification and selection of studies for the review. Adapted from Moher et al. ${ }^{31}$ PRISMA, Preferred Reporting Items for Systematic Reviews andMeta-Analyses; RCT, randomised controlled trial.

\section{Study quality}

PEDro scale total scores ranged from 4 to 7 , indicating that the studies demonstrated fair to high quality (high $=53 \%$, fair $=47 \%$ ) (table 2). Only $21 \%$ of trials reported concealed allocation and $37 \%$ used an intention to treat analysis.

\section{Publication bias}

The funnel plot for the analysis appeared to be roughly symmetrical. Publication bias was tested using Egger's regression method $^{36}$ and the Begg-Mazumdar test, ${ }^{37}$ with a $\mathrm{p}$ value $<0.05$ suggesting the presence of bias. The effect size was not modified by the Duval and Tweedie trim and fill test. Additionally, funnel plots in figure 2 display study specific effect estimates in relation to the SE, assessing the potential presence of publication bias. Heterogeneity was limited $\left(\mathrm{I}^{2}=33 \%, \mathrm{p}=0.11\right)$.

\section{Assessment of overall effect}

Primary outcomes: effect of yoga on depressive symptoms in people with a mental disorder

A moderate effect of yoga on depressive symptoms compared with all control groups was found $(\mathrm{SMD}=-0.41 ; 95 \% \mathrm{CI}-0.65$ to $-0.17, \mathrm{p}<0.001$; heterogeneity: $\mathrm{I}^{2}=50 \% ; \mathrm{Q}=23.86 ; \mathrm{p}=0.02$; $\mathrm{RCT}=13$; yoga $n=333$; control $n=299$ ) (figure 2).

\section{Subgroup analyses \\ Control group}

Subgroup analyses based on control groups revealed evidence for a moderate effect of yoga on depressive symptoms compared with waitlist control $(n=4, S M D=-0.58 ; 95 \% C I-1.03$ to $-0.12, \mathrm{p}<0.05$ ), a small, but not statistically significant, effect compared with treatment as usual $(n=2, S M D=-0.39$; $95 \% \mathrm{CI}-1.14$ to $0.36, \mathrm{p}=0.31)$ and to attention controls $(\mathrm{n}=8$, $\mathrm{SMD}=-0.21 ; 95 \% \mathrm{CI}-0.54$ to $0.12, \mathrm{p}=0.22$ ).

\section{Diagnostic category}

Subgroup analyses based on diagnostic groups revealed evidence for a moderate effect of yoga on depressive symptoms in depressive disorders $(\mathrm{n}=8, \mathrm{SMD}=-0.40 ; 95 \% \mathrm{CI}-0.67$ to $-0.13, \mathrm{p}<0.01)$, no effect in PTSD $(\mathrm{n}=2, \mathrm{SMD}=-0.01 ; 95 \% \mathrm{CI}$, -0.51 to $0.48, \mathrm{p}=0.95$ ), a small, but not statistically significant, effect in alcohol use disorders $(n=1, \mathrm{SMD}=-0.24 ; 95 \% \mathrm{CI}$ -1.40 to $0.92, p=0.69)$ and a large effect in schizophrenia $(\mathrm{n}=2, \mathrm{SMD}=-0.90 ; 95 \% \mathrm{CI}-1.44$ to $-0.35, \mathrm{p}<0.01)$.

Secondary outcomes: effect of yoga on physical health

Insufficient data were available to pool the effects of yoga on physical health outcomes. Seven studies included measures of physical health. One study ${ }^{52}$ examined cardiometabolic health (fasting blood glucose and total cholesterol) and found no significant differences between the yoga and control groups over the 8 week intervention. Another study by the same authors ${ }^{53}$ examined physical fitness (strength) by measuring hand grip strength, lower limb muscle endurance and knee flexion strength to body weight ratio. The yoga group had significantly greater improvements across all three measures compared with the treatment as usual group. Lin et $a l^{57}$ assessed aerobic fitness with a $\mathrm{VO}_{2} \max$ test and found no significant changes between groups. While not statistically significant, Field et $a l^{40}$ reported reduced cortisol levels within a single session (acute response) for both yoga and social support groups, and increased cortisol levels from baseline to last session (chronic response). Three studies measured physical health/functioning with self-report questionnaires. Uebelacker et $a l^{46}$ reported no significant improvements for Short Form Health Survey (SF-20) scores between groups. Buttner et $a l^{39}$ however used the SF-36 to measure health related quality of life and reported that the yoga group experienced a steeper linear increase in scores compared with the waitlist control. Visceglia et $a l^{19}$ reported that the yoga group had significant improvements in the physical health domain of the World Health Organization Quality of Life Instrument (WHOQOL-BREF) whereas no improvement was reported for the control group.

\section{Meta-regression}

Meta-regression analyses using a mixed effects model revealed that number of sessions per week had a significant effect on depressive symptoms, with higher session frequency leading to a greater improvement in symptoms $(\beta=-0.44 ; \mathrm{p}<0.001 ; \mathrm{CI}$ -0.66 to -0.21 ) (Supplementary Table 2). Other intervention variables, such as trial quality, session duration, intervention length, supervision and number of yoga components included (as reported) did not influence depressive symptoms.

\section{Remission rates}

Three studies reported remission rates where symptom severity scores returned to below the diagnostic threshold. van der Kolk et $a l^{49}$ reported that $52 \%$ of participants no longer had clinically significant PTSD symptoms compared with 21\% in the health education control group; Prathikanti, et $a l^{44}$ reported that $60 \%$ of participants reached remission in the yoga group compared with $10 \%$ in the control group; and Uebelacker et al ${ }^{46}$ reported no significant difference in remission rates between the groups at the end of the intervention but at the 3 month follow-up, 39\% of participants in the yoga group were in remission compared with $24 \%$ in the control group.

\section{Adverse events, attendance and dropout rates}

Seven studies reported on adverse events. ${ }^{194446-485354}$ All reported that no adverse events occurred from the intervention. Four studies 


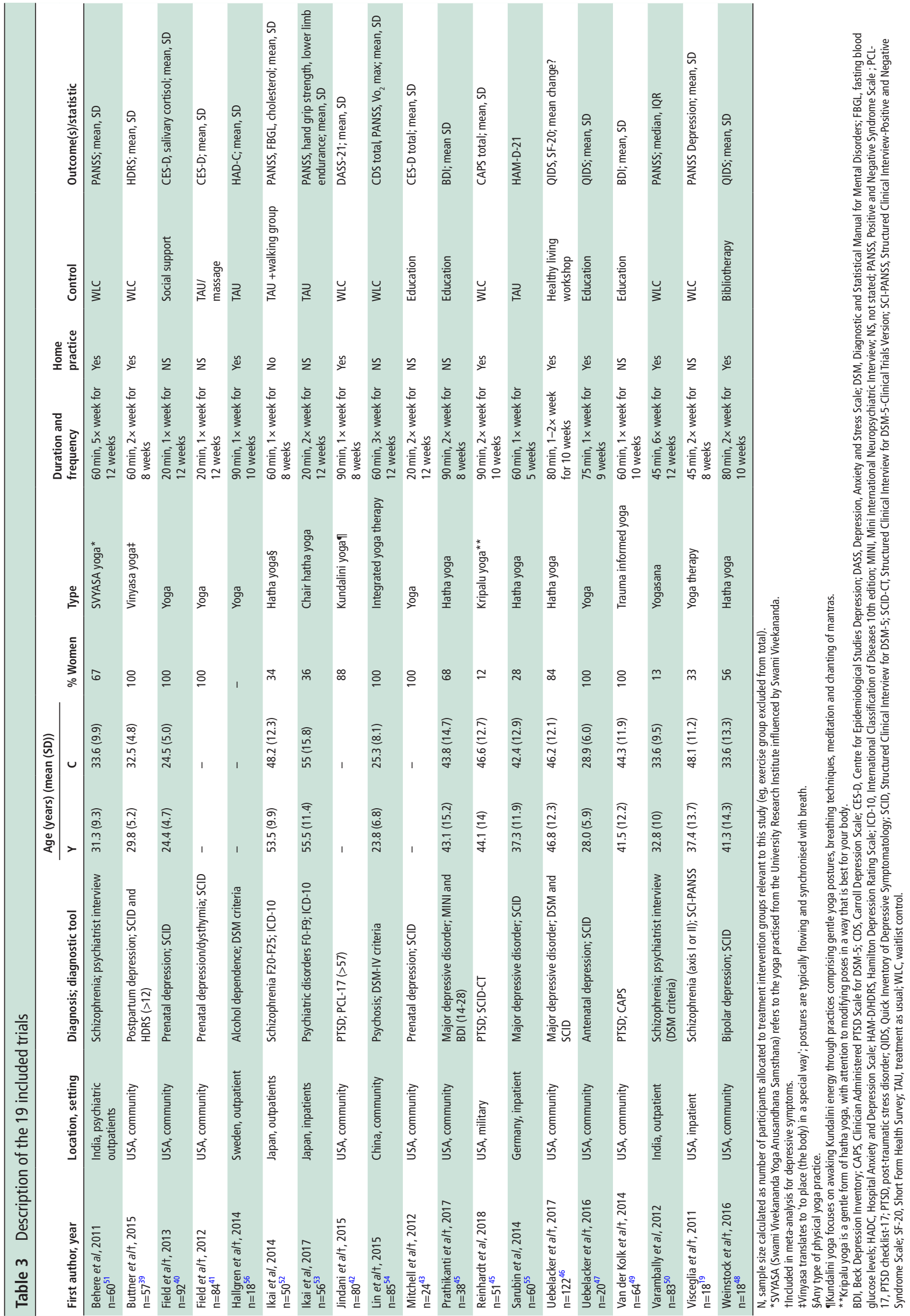




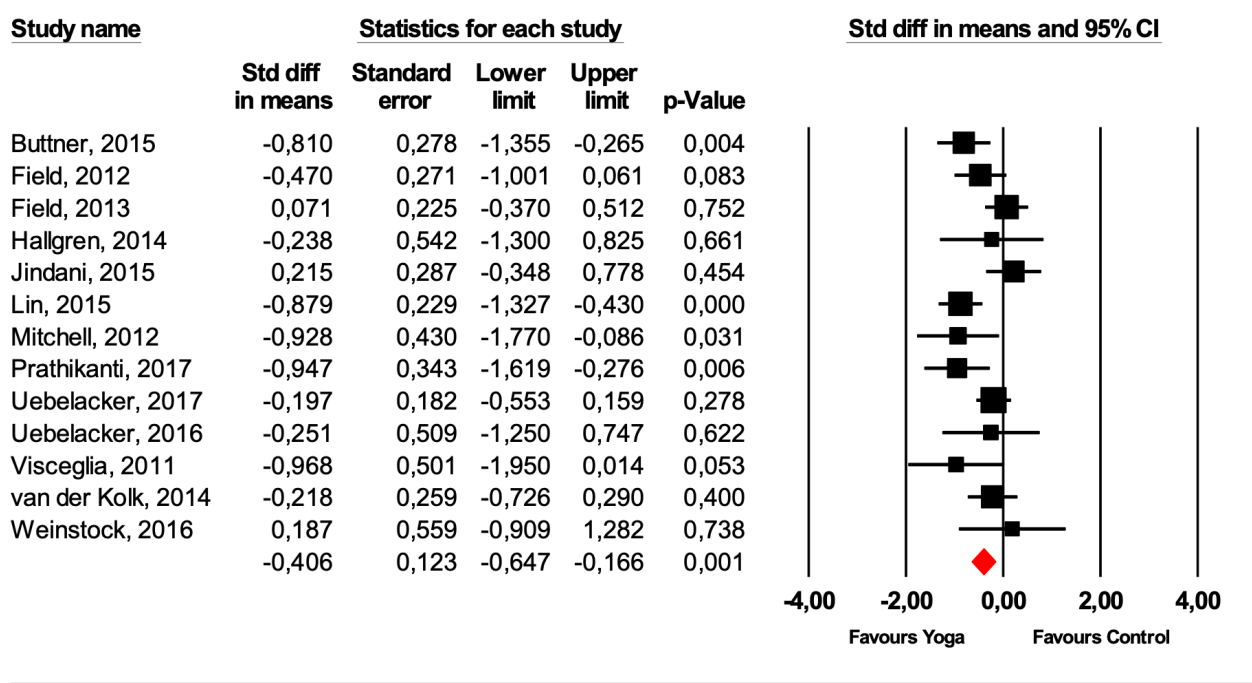

Figure 2 Effects of yoga versus treatment as usual, waitlist control or attention control interventions on depressive symptoms across a range of mental disorders, showing estimates of effect size with 95\% Cls and relative weight (\% weight) for each trial.

reported on some form of intervention fidelity (eg, participant fidelity such as attendance or adherence, or instructor fidelity, such as adherence to intervention protocol) (table 4). ${ }^{44-46-48}$ Dropout rates are reported in table 4 . Total dropout rate ranged from $9 \%$ to $48 \%$. All but two studies reported dropout rates. ${ }^{1943}$

\section{DISCUSSION}

The purpose of this systematic review and meta-analysis was to examine the effects that a predominantly physical yoga practice has on depressive symptoms in people with a diagnosed mental disorder. The results demonstrated that yoga has a moderate effect on reducing depressive symptoms across a range of diagnosed mental disorders, thus suggesting yoga may be a viable transdiagnostic intervention for the management of depressive symptoms in people with mental disorders.
The magnitude of the effect of yoga on depressive symptoms compared with treatment as usual, waitlist and attention control combined $(\mathrm{SMD}=-0.41)$ was comparable with findings in previous meta-analyses of yoga for depressive symptoms in people with depressive disorders (Cramer et al, ${ }^{28} \mathrm{SMD}=-0.69$, $95 \% \mathrm{CI}=-0.99$ to 0.39 ; Gong et al, $\mathrm{SMD}=-0.59$, $95 \% \mathrm{CI}=-0.94$ to -0.25$)$. Gong and colleagues ${ }^{60}$ included six trials in their meta-analysis, of which four were also included in the current review. Cramer and colleagues included nine trials in their meta-analysis, of which four were also included in the current review. The variance in results is likely a reflection of the larger sample size with additional studies of high methodological quality, resulting in a more conservative effect. However, the current review has reported the most precise estimate so far, as indicated by narrower confidence intervals. Subgroup analyses

Table 4 Dropout, attendance and adverse events of the included studies

\begin{tabular}{|c|c|c|c|}
\hline Study & Dropout & Intervention fidelity & Adverse events \\
\hline Behere et $a l^{51}$ & Total $18 \%$ ( $20 \%$ yoga; $15 \%$ control) & - & - \\
\hline Buttner et $a^{39}$ & Total $11 \%$ (14\% yoga; $7 \%$ control) & - & - \\
\hline Field et $a l^{40}$ & Total $14 \%$ & - & - \\
\hline Field et $a l^{41}$ & Total $25 \%$ & - & - \\
\hline Hallgren et $a^{56}$ & Total $22 \%$ (33\% yoga; $11 \%$ control) & - & - \\
\hline Ikai et $\left.a\right|^{52}$ & Total $28 \%$ & - & - \\
\hline Lin et al, $2015^{54}$ & Total $23.40 \%$ & - & 0 \\
\hline Mitchell et $a l^{43}$ & - & - & - \\
\hline Prathikanti et al ${ }^{44}$ & Total $44 \%$ & Total $65 \%$ adherence rate $(74 \%$ yoga; $51 \%$ control) & 0 \\
\hline Reinhardt et $\mathrm{al}_{\mathrm{a}}{ }_{\mathrm{a}}$ & Total $48 \%$ & - & - \\
\hline Sarubin et $a l^{55}$ & Total $12 \%$ & - & - \\
\hline Varambally et al ${ }^{50}$ & Total $20 \%$ & - & - \\
\hline Visceglia et $a /^{19}$ & - & - & 0 \\
\hline Weinstock et $a l^{48}$ & Total $22 \%$ & Average 4.8 (SD 5.12) classes attended/10 $\mathrm{min}(20 \mathrm{max}$ ) & 0 \\
\hline
\end{tabular}


in the reviews of both Gong et al (2015) and Cramer et al (2013) indicated larger effects on depressive symptoms for meditation based yoga interventions compared with physically active yoga interventions. This may partially explain the smaller overall effect seen in the present study, as only physically active yoga interventions were included. While the present review yields a smaller result than physical activity reviews, a moderate effect size indicates that yoga is an efficacious modality of physical activity that should be considered when offering interventions to psychiatric populations, particularly considering the importance of preference and enjoyment for long term adherence and behaviour change. ${ }^{61}$ Determining the benefits of mindful exercise, such as yoga, against conventional exercise should be explored further. Preliminary data suggest that combining mindfulness with conventional exercise is effective for reducing depressive symptoms. ${ }^{62}$ There is strong evidence to support that both mindfulness ${ }^{21}$ and aerobic and/or resistance exercise $^{1463}$ independently reduce depressive symptoms. Exploring whether the effects are strengthened when these are combined, or whether movement allows for greater engagement in mindfulness for novices, is a novel and integral topic. Identifying the mechanisms by which yoga may reduce depressive symptoms is of clinical importance yet is beyond the scope of this review. Nonetheless, a recent comprehensive review ${ }^{64}$ of the potential mechanisms by which physical activity may reduce depressive symptoms identified a range of biopsychosocial pathways which are likely to have broad relevance to understanding the mechanisms of yoga.

Extrapolating an effective, and even optimal 'dose' of yoga to facilitate clinically significant change would help inform clinical practice. Further exploration of yoga in comparison with conventional or purposefully non-mindful exercise may help determine effective components of yoga practice. In order to explore a dose-response relationship between yoga practice and the longevity of its benefits, future research should consider collecting follow-up data at varying time points postintervention. Only six included articles reported follow-up data, with time points ranging from 12 weeks ( 2 weeks post intervention), ${ }^{46} 16$ weeks (8 weeks post intervention), ${ }^{51}{ }^{52} 18$ weeks ( 6 weeks post intervention), ${ }^{53} 6$ months (14 weeks post intervention) $)^{4656}$ and 18 months (15 months post intervention). ${ }^{54}$ Therefore, conclusions about the potential long term effects of yoga on depressive symptoms were unable to be made.

We found that the number of sessions per week moderated the effect of yoga on depressive symptoms. This is an important finding and should be considered in the design of future yoga interventions targeted at depressive symptoms in people with mental disorders. Interventions should aim to increase the frequency of their sessions per week, as opposed to the duration of each session or the overall duration of the intervention. Further investigation into the duration of effects, and whether particular types of yoga elicit better effects, would be a valuable research question to explore.

Structured exercise in depression and schizophrenia has been shown to have better outcomes when delivered by a qualified health professional. ${ }^{65}{ }^{66}$ However, the impact of qualification in yoga practice and outcomes is unclear. The qualification of yoga practitioners who delivered the interventions in the included RCTs was not reported. Currently, yoga teacher training and education courses are not regulated or standardised, nor accredited within any institutes of higher education. ${ }^{67}$ This may have implications when dealing with clinical populations who have chronic and complex conditions that require indepth understanding of pathology and psychopathology as well as contraindications and safe prescription of exercise. Furthermore, additional training and upskilling in mental health first aid should be considered for any exercise professional or yoga practitioner delivering interventions for this population. Future research should report the qualification level and type, as well as the experience of the yoga practitioner delivering the intervention, and further analyses should explore the effect this has on intervention outcomes/effectiveness.

Along with efficacy, this review also demonstrated the feasibility of yoga for people with mental disorders. Attendance and dropout did not differ significantly from control groups, with some studies reporting better adherence to yoga than control condition. ${ }^{465051}$ A common barrier included 'difficulties making it to classes' ${ }^{48}$ thus having yoga services available within inpatient and outpatient mental health facilitates may address this barrier and is likely to have positive effects on symptoms of depression, as well as increasing physical activity levels and promoting greater physical health.

\section{Limitations}

The present review included a range of yoga interventions but due to the relatively small number of trials identified and the poorly defined interventions, it was not possible to conduct separate analyses based on the type and intensity of the interventions. Results from the control and diagnostic category subgroup analyses must also be interpreted with caution because of the small number of studies included. This review did not look at comparisons with true active bona fide interventions and only a small number of studies were available for each kind of control group, thus limiting the comparability of yoga against other interventions for depressive symptoms.

This review has limitations that relate to the primary trials included, such as non-standardised reporting of intervention protocols, lack of intervention description (eg, type of yoga and components of yoga prescribed), poor methodological quality (eg, inadequate blinding of assessors, inadequate follow-up, lack of intention to treat analysis), mix of interviewer rated and selfreport outcome measures, and lack of reporting intervention adherence and adverse events. More high quality studies with sufficient sample sizes based on predetermined power analyses are required.

It is essential that future yoga research is reported in a standardised manner whereby the components of yoga can be clearly determined; mindfulness, meditation and physical activity. For further understanding of the mechanism by which yoga has an effect on mental and physical health, intervention variables such as type of yoga, intensity, environment, instructor qualification, specific postures, cueing, philosophical focuses, mindfulness techniques and breathing techniques should be adequately reported. Additionally, the question of longevity of the effects of yoga following intervention cessation requires further investigation; future studies should include long term follow-up. The effects of yoga may be moderated by participant specific factors, such as comorbidities and time since diagnosis, and therefore these factors warrant reporting and investigation in future studies.

Meaningful measures of physical health should be measured so that yoga intervention data can be compared with other modalities of physical activity and independently examined with regard to its physical health benefits, particularly among individuals with a mental disorder. 


\section{CONCLUSION}

This review found evidence of a positive effect of yoga beyond usual care for reducing depressive symptoms in people with a range of mental disorders. There was a dose-response relationship between the number of yoga sessions per week and improvements in depressive symptoms. Consideration of yoga as an evidence based exercise modality alongside conventional forms of exercise is warranted given the positive results of this review. Yoga may provide an additional or alternative strategy to engage people experiencing depression in meaningful physical activity.

\section{What is already known?}

- There is an urgent need to improve physical and mental health for people with diagnosed mental disorders. This is a priority for clinicians, healthcare services, researchers and policy makers.

- There is evidence to support exercise and mindfulness as singular therapies for improving depressive symptoms.

\section{What are the new findings?}

- Physically active yoga has a moderate positive effect on improving depressive symptoms in people with a range of mental disorders.

- Session frequency per week significantly influences the magnitude of depressive symptom reduction.

\section{Author affiliations}

Alliance for Research in Exercise, Nutrition and Activity, University of South Australia: Allied Health and Human Performance, Adelaide, South Australia, Australia 'Department of Sports Methods and Techniques, Federal University of Santa Maria, Santa Maria, Brazil

${ }^{3}$ Keeping the Body In Mind, South Eastern Sydney Local Health District, Sydney, New South Wales, Australia

${ }^{4}$ School of Psychology, Social Work and Social Policy, University of South Australia, Adelaide, South Australia, Australia

${ }^{5}$ Institute of Psychiatry, Psychology and Neuroscience, King's College London, London, UK

${ }^{6}$ Division of Psychology and Mental Health, University of Manchester, Manchester, UK ${ }^{7}$ School of Psychiatry, University of New South Wales, Sydney, New South Wales, Australia

${ }^{8}$ Black Dog Institute, Randwick, New South Wales, Australia

Twitter Jacinta Brinsley @theyogidoc_and Simon Rosenbaum @simon_rosenbaum

Contributors JB and SR conceived the study. OL screened papers. FS, BS and JF assisted with data analysis. All authors contributed to data interpretation and drafting of the manuscript and approved the final version.

Funding Simon Rosenbaum is funded by an NHMRC Fellowship APP1123336. Brendon Stubbs is supported by a Clinical Lectureship (ICA-CL-2017-03-001) jointly funded by Health Education England (HEE) and the National Institute for Health Research (NIHR). Brendon Stubbs is part funded by the NIHR Biomedical Research Centre at South London and Maudsley NHS Foundation Trust. The views expressed are those of the author(s) and not necessarily those of the (partner organisation), the NHS, the NIHR or the Department of Health and Social Care. Joseph Firth is supported by a University of Manchester Presidential Fellowship.

Competing interests None declared.

Patient consent for publication Not required.

Provenance and peer review Not commissioned; externally peer reviewed.

ORCID iDs

Jacinta Brinsley http://orcid.org/0000-0002-2588-9649

Simon Rosenbaum http://orcid.org/0000-0002-8984-4941

\section{REFERENCES}

1 Vigo D, Thornicroft G, Atun R. Estimating the true global burden of mental illness. Lancet Psychiatry 2016;3:171-8.
2 Greden JF. The burden of recurrent depression: causes, consequences, and future prospects. J Clin Psychiatry 2001;62(Suppl 22):5-9.

3 Murray CJ, Lopez AD, World Health Organization. The global burden of disease: a comprehensive assessment of mortality and disability from diseases, injuries, and risk factors in 1990 and projected to 2020: summary, 1996.

4 Rebar AL, Stanton R, Rosenbaum S. Comorbidity of depression and anxiety in exercise research. Lancet Psychiatry 2017;4:519.

5 Buckley PF, Miller BJ, Lehrer DS, et al. Psychiatric comorbidities and schizophrenia. Schizophr Bull 2009;35:383-402.

6 Royal Australian New Zealand College of Psychiatrists. The economic cost of serious mental illness and comorbidities in Australia and New Zealand. Melbourne RANZCP; 2016.

7 Vancampfort D, Stubbs B, Mitchell AJ, et al. Risk of metabolic syndrome and its components in people with schizophrenia and related psychotic disorders, bipolar disorder and major depressive disorder: a systematic review and meta-analysis. World Psychiatry 2015;14:339-47.

8 Vancampfort D, Firth J, Schuch FB, et al. Sedentary behavior and physical activity levels in people with schizophrenia, bipolar disorder and major depressive disorder: a global systematic review and meta-analysis. World Psychiatry 2017:16:308-15.

9 Farmer ME, Locke BENZ, MOŚCICKI EVEK, et al. Physical activity and depressive symptoms: the NHANES I epidemiologic follow-up study. Am J Epidemiol 1988;128:1340-51.

10 Schuch F, Vancampfort D, Firth J, et al. Physical activity and sedentary behavior in people with major depressive disorder: a systematic review and meta-analysis. J Affect Disord 2017;210:139-50.

11 Stubbs B, Vancampfort D, Hallgren M, et al. EPA guidance on physical activity as a treatment for severe mental illness: a meta-review of the evidence and position statement from the European Psychiatric Association (EPA), supported by the International Organization of Physical Therapists in Mental Health (IOPTMH). Eur Psychiatry 2018;54:124-44.

12 Firth J, Siddiqi N, Koyanagi A, et al. The Lancet psychiatry Commission: a blueprint for protecting physical health in people with mental illness. Lancet Psychiatry 2019:6:675-712.

13 Teasdale SB, Ward PB, Rosenbaum S, et al. Solving a weighty problem: systematic review and meta-analysis of nutrition interventions in severe mental illness. $\mathrm{Br} J$ Psychiatry 2017;210:110-8.

14 Schuch FB, Vancampfort D, Richards J, et al. Exercise as a treatment for depression: a meta-analysis adjusting for publication bias. J Psychiatr Res 2016;77:42-51.

15 National Mental Health Commission. Equally well consensus statement: improving the physical health and wellbeing of people living with mental illness in Australia. Sydney, Australia, 2016.

16 Malhi GS, Bassett D, Boyce P, et al. Royal Australian and New Zealand College of Psychiatrists clinical practice guidelines for mood disorders. Aust N Z J Psychiatry 2015:49:1087-206.

17 Kennedy SH, Lam RW, McIntyre RS, et al. Canadian network for mood and anxiety treatments (CANMAT) 2016 clinical guidelines for the management of adults with major depressive disorder: section 3. pharmacological treatments. Can J Psychiatry 2016:61:540-60.

18 Schuch FB, Morres ID, Ekkekakis P, et al. Exercise works for depression: bridging the implementation gap and making exercise a core component of treatment. Acta Neuropsychiatr 2017;29:124-6.

19 Visceglia E, Lewis S. Yoga therapy as an adjunctive treatment for schizophrenia: a randomized, controlled pilot study. J Altern Complement Med 2011;17:601-7.

20 de Manincor M, Bensoussan A, Smith C, et al. Establishing key components of yoga interventions for reducing depression and anxiety, and improving well-being: a Delphi method study. BMC Complement Altern Med 2015;15:85.

21 Hofmann SG, Sawyer AT, Witt AA, et al. The effect of mindfulness-based therapy on anxiety and depression: a meta-analytic review. J Consult Clin Psychol 2010;78:169-83.

22 Gotink RA, Chu P, Busschbach JJV, et al. Standardised mindfulness-based intervention in healthcare: an overview of systematic reviews and meta-analyses of RCTs. PLoS One 2015;10:e0124344.

23 Ussher M, Stanbury L, Cheeseman V, et al. Physical activity preferences and perceived barriers to activity among persons with severe mental illness in the United Kingdom. Psychiatr Serv 2007;58:405-8

24 Siris SG. Depression in schizophrenia: perspective in the era of "atypical" antipsychotic agents. Am J Psychiatry 2000;157:1379-89.

25 Brown TA, Campbell LA, Lehman $\mathrm{CL}$, et al. Current and lifetime comorbidity of the DSM-IV anxiety and mood disorders in a large clinical sample. J Abnorm Psychol 2001:110:585-99.

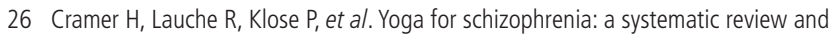
meta-analysis. BMC Psychiatry 2013;13:32.

27 Kirkwood G, Rampes H, Tuffrey $\mathrm{V}$, et al. Yoga for anxiety: a systematic review of the research evidence. Br J Sports Med 2005:39:884-91.

28 Cramer $\mathrm{H}$, Lauche $\mathrm{R}$, Langhorst J, et al. Yoga for depression: a systematic review and meta-analysis. Depress Anxiety 2013;30:1068-83.

29 Pilkington K, Kirkwood G, Rampes $\mathrm{H}$, et al. Yoga for depression: the research evidence J Affect Disord 2005;89:13-24 
30 Uebelacker LA, Epstein-Lubow G, Gaudiano BA, et al. Hatha yoga for depression: critical review of the evidence for efficacy, plausible mechanisms of action, and directions for future research. J Psychiatr Pract 2010;16:22-33.

31 Moher D, Liberati A, Tetzlaff J, et al. Preferred reporting items for systematic reviews and meta-analyses: the PRISMA statement. PLoS Med 2009;6:e1000097.

32 American Psychiatric Association. Diagnostic and statistical manual of mental disorders (DSM-5®. Washington, DC: American Psychiatric Pub, 2013.

33 Honey EP. I shrunk the pooled SMD! Guide to critical appraisal of systematic reviews and meta-analyses using the Cochrane review on exercise for depression as example. Ment Health Phys Act 2015:8:21-36.

34 Maher CG, Sherrington C, Herbert RD, et al. Reliability of the PEDro scale for rating quality of randomized controlled trials. Phys Ther 2003;83:713-21.

35 The Cochrane CollaborationHiggins JPT, Green S, eds. Cochrane Handbook for systematic reviews of interventions version 5.1.0, 2011. www.handbook.cochrane.org

36 Egger M, Davey Smith G, Schneider M, et al. Bias in meta-analysis detected by a simple, graphical test. BMJ 1997;315:629-34.

37 Begg CB, Mazumdar M. Operating characteristics of a RANK correlation test for publication bias. Biometrics 1994;50:1088-101.

38 Duval S, Tweedie R. Trim and fill: a simple Funnel-plot-based method of testing and adjusting for publication bias in meta-analysis. Biometrics 2000;56:455-63.

39 Buttner MM, Brock RL, O'Hara MW, et al. Efficacy of yoga for depressed postpartum women: a randomized controlled trial. Complement Ther Clin Pract 2015;21:94-100.

40 Field T, Diego M, Delgado J, et al. Yoga and social support reduce prenatal depression, anxiety and cortisol. J Bodyw Mov Ther 2013;17:397-403.

41 Field T, Diego M, Hernandez-Reif M, et al. Yoga and massage therapy reduce prenatal depression and prematurity. J Bodyw Mov Ther 2012;16:204-9.

42 Jindani F, Turner N, Khalsa SBS. A yoga intervention for posttraumatic stress: a preliminary randomized control trial. Evid Based Complement Alternat Med 2015;2015:351746:1-8.

43 Mitchell J, Field T, Diego M, et al. Yoga reduces prenatal depression symptoms. Psychology 2012;3:782-6.

44 Prathikanti S, Rivera R, Cochran A, et al. Treating major depression with yoga: a prospective, randomized, controlled pilot trial. PLoS One 2017;12:e0173869.

45 Reinhardt KM, Noggle Taylor JJ, Johnston J, et al. Kripalu yoga for military veterans with PTSD: a randomized trial. J Clin Psychol 2018;74:93-108.

46 Uebelacker LA, Tremont G, Gillette LT, et al. Adjunctive yoga V. health education for persistent major depression: a randomized controlled trial. Psychol Med 2017;47:2130-42.

47 Uebelacker LA, Battle CL, Sutton KA, et al. A pilot randomized controlled trial comparing prenatal yoga to perinatal health education for antenatal depression. Arch Womens Ment Health 2016;19:543-7.

48 Weinstock LM, Broughton MK, Tezanos KM, et al. Adjunctive yoga versus bibliotherapy for bipolar depression: a pilot randomized controlled trial. Ment Health Phys Act 2016;11:67-73.

49 van der Kolk BA, Stone L, West J, et al. Yoga as an adjunctive treatment for posttraumatic stress disorder: a randomized controlled trial. J Clin Psychiatry 2014;75:e559-65.

50 Varambally S, Gangadhar BN, Thirthalli J, et al. Therapeutic efficacy of addon yogasana intervention in stabilized outpatient schizophrenia: randomized controlled comparison with exercise and waitlist. Indian J Psychiatry 2012;54:227-32

51 Behere RV, Arasappa R, Jagannathan A, et al. Effect of yoga therapy on facial emotion recognition deficits, symptoms and functioning in patients with schizophrenia. Acta Psychiatr Scand 2011;123:147-53.

52 Ikai S, Suzuki T, Uchida H, et al. Effects of weekly one-hour hatha yoga therapy on resilience and stress levels in patients with schizophrenia-spectrum disorders: an eight-week randomized controlled trial. J Altern Complement Med 2014;20:823-30.
53 Ikai S, Uchida H, Mizuno Y, et al. Effects of chair yoga therapy on physical fitness in patients with psychiatric disorders: a 12-week single-blind randomized controlled trial. J Psychiatr Res 2017;94:194-201.

54 Lin J, Chan SK, Lee EH, et al. Aerobic exercise and yoga improve neurocognitive function in women with early psychosis. NPJ Schizophr 2015;1:15047.

55 Sarubin N, Nothdurfter C, Schüle C, et al. The influence of hatha yoga as an add-on treatment in major depression on hypothalamic-pituitary-adrenal-axis activity: a randomized trial. J Psychiatr Res 2014;53:76-83.

56 Hallgren M, Romberg K, Bakshi A-S, et al. Yoga as an adjunct treatment for alcohol dependence: a pilot study. Complement Ther Med 2014;22:441-5.

57 JJX L, EHM L, Chang WC, et al. Aerobic exercise and yoga hold promises for improving neuro-cognition and symptom in early psychosis. Schizophr Bull 2015;1:\$320.

58 Mitchell KS, Dick AM, DiMartino DM, et al. A pilot study of a randomized controlled trial of yoga as an intervention for PTSD symptoms in women. J Trauma Stress 2014;27:121-8.

59 Lin S-L, Huang C-Y, Shiu S-P, et al. Effects of yoga on stresS, stress adaption, and heart rate variability among mental health professionals-a randomized controlled trial. Worldviews Evid Based Nurs 2015;12:236-45.

60 Gong H, Ni C, Shen X, et al. Yoga for prenatal depression: a systematic review and meta-analysis. BMC Psychiatry 2015;15:14.

61 Ekkekakis $P$. The dual-mode theory of affective responses to exercise in metatheoretical context: I. initial impetus, basic postulates, and philosophical framework. Int Rev Sport Exerc Psychol 2009;2:73-94.

62 Alderman BL, Olson RL, Brush CJ, et al. MAP training: combining meditation and aerobic exercise reduces depression and rumination while enhancing synchronized brain activity. Trans/ Psychiatry 2016;6:e726.

63 Hoffman BM, Babyak MA, Craighead WE, et al. Exercise and pharmacotherapy in patients with major depression: one-year follow-up of the SMILE study. Psychosom Med 2011;73:127-33.

64 Kandola A, Ashdown-Franks G, Hendrikse J, et al. Physical activity and depression: towards understanding the antidepressant mechanisms of physical activity. Neurosci Biobehav Rev 2019;107:525-39.

65 Vancampfort D, Stubbs B, Sienaert P, et al. What are the factors that influence physical activity participation in individuals with depression? A review of physical activity correlates from 59 studies. Psychiatr Danub 2015;27:210.

66 Stubbs B, Vancampfort D, Rosenbaum S, et al. Dropout from exercise randomized controlled trials among people with depression: a meta-analysis and meta regression. J Affect Disord 2016;190:457-66.

67 National Assessment and Accreditation Council. The global yoga accreditation Summit. New York, 2019.

68 Balasubramaniam M, Telles S, Doraiswamy PM. Yoga on our minds: a systematic review of yoga for neuropsychiatric disorders. Front Psychiatry 2012;3:117.

69 Broderick J, Knowles A, Chadwick J, et al. Yoga versus standard care for schizophrenia Cochrane Database Syst Rev 2015:CD010554.

70 Cramer H, Anheyer D, Lauche R, et al. A systematic review of yoga for major depressive disorder. J Affect Disord 2017;213:70-7.

71 Cramer H, Anheyer D, Saha FJ, et al. Yoga for posttraumatic stress disorder - a systematic review and meta-analysis. BMC Psychiatry 2018;18:72.

72 Cramer H, Lauche R, Anheyer D, et al. Yoga for anxiety: a systematic review and metaanalysis of randomized controlled trials. Depress Anxiety 2018;35:830-43.

73 da Silva TL, Ravindran LN, Ravindran AV. Yoga in the treatment of mood and anxiety disorders: a review. Asian J Psychiatr 2009;2:6-16.

74 Gallegos AM, Crean HF, Pigeon WR, et al. Meditation and yoga for posttraumatic stress disorder: a meta-analytic review of randomized controlled trials. Clin Psychol Rev 2017:58:115-24.

75 Vancampfort D, Vansteelandt K, Scheewe T, et al. Yoga in schizophrenia: a systematic review of randomised controlled trials. Acta Psychiatr Scand 2012;126:12-20. 